\title{
Broken Symmetries, Random Morphogenesis, and Biometric Distance
}

\author{
John Daugman, Cathryn Downing
}

\begin{abstract}
This paper discusses the role of symmetry-breaking in biometric recognition. Using publicly available databases, we investigate three kinds of broken symmetries in iris patterns: binocular, monocular, and monozygotic. We report a small but statistically significant difference in similarities between the ipsilateral and the contralateral eyes of twins, and also between genetically identical and nonidentical eyes. Another new finding is a doubling in the variance of Hamming distance scores under a simple monocular mirror transformation, which is consistent with an assessment of entropy.
\end{abstract}

Index Terms-Morphogenesis, biometric distance, iris, twins.

\section{INTRODUCTION}

I NTER-PERSON variation is the basis of biometric discriminability, and its power to avoid identity collisions is determined by the entropy inherent in the biometric patterns. Entropy describes the randomness and complexity of their variation in terms of the number of possible states and their relative probability distribution. Symmetries reduce entropy, because they imply that one part of a pattern is predicted by another part. A simple example is the bilateral symmetry normally seen in a face in frontal view. If one half of the face is predictable from the other half by simple mirror reflection, then no additional information is obtained from the second half. More generally in information theory [1], if a random variable $X$ is any deterministic function of another random variable $Y$, then the conditional entropy of $X$ given $Y$ is $H(X \mid Y)=0$. Conversely if an expected symmetry is broken - for example a pronounced facial asymmetry, or gait with a limp - then this can be an important biometric signal.

Symmetry breaking creates complexity [2], and thereby it increases entropy. Embryological morphogenesis proceeds through a sequence of symmetry breaking steps, starting from equivalent cells in a spherical blastula, differentiating into nonequivalent layers of cells in a gastrula, then forming distinct anterior and posterior poles, and leading ultimately to the full anatomical complexity of an organism. The morphogenesis of spontaneous complex properties in biological annular structures is an important topic in the study of phyllotaxis [3]. This paper documents three forms of broken symmetry and the complexity it creates in iris patterns, with resulting effects on discriminability. Two of the symmetries investigated are in geometrical domains, and a third set involves the domain of genetic expression.

Biometric features lie on a continuum whose two endpoints are genotypic (traits that are fully determined genetically),

Department of Computer Science and Technology, Cambridge University, 15 J.J. Thomson Avenue, Cambridge CB3 OFD UNITED KINGDOM. e-mail: John.Daugman@CL.cam.ac.uk, Cathryn.Downing@CL.cam.ac.uk
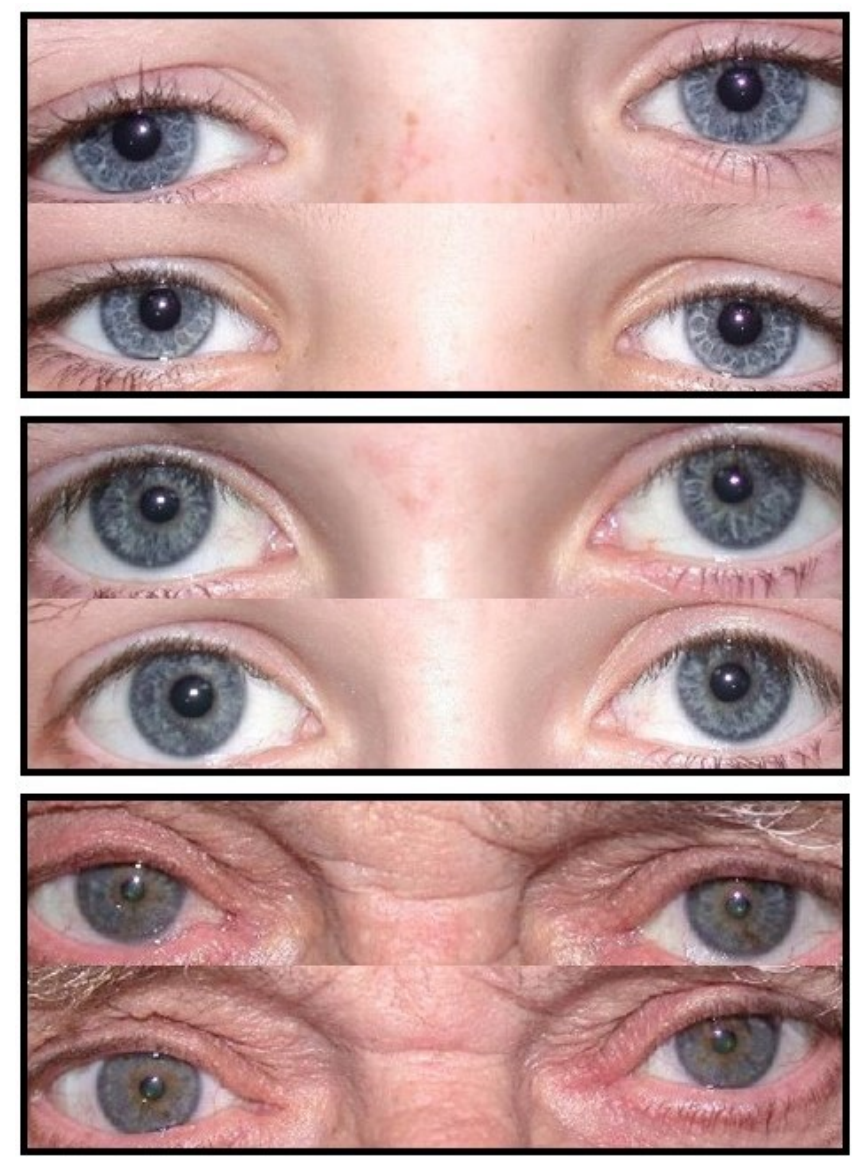

Fig. 1. Monozygotic twins. Courtesy of Melanogenix Laboratory, Australia. Iris symmetries are broken both contra- and ipsi-laterally, but not equally.

and phenotypic (traits that are not). (The latter were termed epigenetic until 2009 when the definition of that term was officially changed by a conference, to imply heritability [4] despite such traits not being encoded in the genome itself.) Examples of the former traits include sex, blood group, and eye colour; examples of the latter include iris patterns and fingerprint minutiae generally. An example in the middle of this continuum is facial appearance: monozygotic (MZ or "identical") twins often look indistinguishable from each other; yet like all faces, their appearance changes over time with age. Curiously, MZ twins tend to track each other as they age. This leads to the striking paradox that MZ twins look more like each other, at any given shared age, than they look like themselves at either earlier or later points in time.

One in about 80 human pregnancies produces twins. Of these, two-thirds are dizygotic (DZ) twins, who (like ordinary 
full siblings) share $25 \%$ of their genes. The other one-third are monozygotic, sharing $100 \%$ of their genes. Thus one pregnancy in about 240 produces two genetically identical persons, so nearly $1 \%$ of all persons (1 in 120) have an MZ twin. This rate sets a lower bound on biometric error rates for face recognition algorithms, as they are completely unable to distinguish between $\mathrm{MZ}$ twins, and even between most DZ twins [5]. The fact that DZ twins are matched in age might make them less distinguishable than ordinary full siblings, despite both cases sharing the same percentage $(25 \%)$ of genes. By contrast, in this paper we document several different forms of symmetry breaking even in the case of genetically identical eyes, twin or one person, which allow robust discrimination across all permutations. We find a very small but statistically significant difference between ipsilateral and contralateral iris comparisons in twins, and between contralateral iris comparisons for the same person and nonrelated persons. We also contrast the requirements of wavelet approaches to classification decisions about similarity, versus identification decisions.

\section{RELATED LITERATURE}

Fingerprint similarity for twins has been extensively studied. Jain et al. (2002) [6] found that MZ twins could be successfully distinguished by their fingerprints but "with a slightly lower accuracy than non-twins." Specifically, when using a threshold that yields a False non-Match Rate of FnMR $=3.5 \%$, non-twins had FMR $=0.3 \%$ while twins had FMR $=1 \%$, or about three times higher. These authors also remarked that: "Dermatoglyphics studies show that there is a high class/type similarity in the fingerprints of identical twins." The MZ fingerprint accuracy reduction was later confirmed by Sun et al. (2010) [7] and Srihari et al. (2008) [8] for 298 pairs of twins, but they reported that DZ twins showed the same loss of fingerprint discriminability as $\mathrm{MZ}$ twins. For face recognition, the failure of algorithms to distinguish MZ twins [5] [9] [10] is worse than the problem for human observers [11].

It is well-known that genetically identical eyes (whether those of MZ twins, or the pair possessed by one person) have similar appearance in terms of colour and general texture. ${ }^{1}$ These similarities were reliably detectable by human observers in perceptual experiments by Larsson et al. (2003) [12] rating the frequency of five types of features, and re-confirmed by Hollingsworth et al. (2010) [13] who showed that untrained human judges could classify iris images as being those of twins with at least $81 \%$ accuracy. Of course the usual goal of any biometric system is to detect individuality, not similarity between different persons, regardless of genetics. But the new field called "family-tree forensics" does aim to detect such similarity between different persons and to exploit genetic relatedness for solving crimes, and in 2019 it was claimed [14] that at least 50 cold cases had thereby been resolved.

\footnotetext{
${ }^{1}$ In the rare cases of heterochromia iridis (https://en.wikipedia.org/wiki/ Heterochromia_iridum), affecting about one person in 1,500 the two eyes may have different colours. Variation of pigment distribution within an iris is more common and is seen, for example, in the elderly pair of twins in Fig. 1 (bottom panel) or indeed in the eyes of Sharbat Gula, the Afghan girl.
}

The genetic basis of eye colour has long been studied, and recent research [15] confirms that eye colour can be predicted accurately from a person's genotype although not in a simple Mendellian fashion. More than 2,000 genes are expressed in the iris [16]. The PAX6 gene controls development of both brain and eye, and indeed aniridia (non-development of iris) results if PAX6 is not expressed; but gene polymorphisms associated with the FOXC gene [15] and a number of modifier loci (OCA2) are involved in determining pigmentation. Besides colour, some general aspects of the "textural" appearance of the iris (such as the presence of crypts, nevi, nodules, and contraction furrows) are also influenced by PAX6 gene expression and are heritable characteristics. This is apparent in the Fig. 1 images and presumably underlies the demonstrations [12] [13] that untrained human observers can reliably classify eyes as twinned. Even a person's biogeographic ancestry (BGA, a population-level variation) as revealed by genomic markers has been shown [16] to be well correlated with iris colour and texture traits, in three variably admixed populations, using human scorers of iris features.

Using iris recognition algorithms, the distinguishability or individuality among genetically identical eyes has not been extensively quantified, nor have all forms of the question been explored. Preliminary studies using small databases [17] [7] found that iris recognition algorithms were not tricked when genetically identical eyes were compared, despite similarities that are easily perceived by human observers [13]. Clearly the various genes expressed in the iris do affect its general appearance in terms of colour, frequency of specific features, and overall texture; but there remains a large degree of randomness in the detailed iris morphogenesis, easily allowing algorithms to make sharp distinctions of identity.



Fig. 2. Demodulation-based encoding of image structure by even- and oddsymmetric phasor modules, resolving both local amplitude $A(x, y)$ and phase $\phi(x, y)$ descriptions. From Fig. 14, Daugman and Downing (1995) [18].

For algorithms using complex (e.g. Gabor) wavelets, it is noteworthy that the two tasks of: (1) iris classification or association based on general textural appearance, and (2) iris identification based on detailed phase sequence, can both be performed by the same set of quadrature encoding units, if their outputs are resolved into: (1) modulus energy or power, and (2) phase structure. This is illustrated in Fig. 2. Evensymmetric and odd-symmetric encoder "receptive fields" are spatially coincident in each pair, and may be regarded as the 
product of an elliptical Gaussian times a cosine wave or a sine wave. Patches of an iris image $I(x, y)$ are projected onto such phasor modules. Emerging from the top of each such module is the sum of squares of these inner products, $A^{2}(x, y)$, which is a power measure because of the quadrature relationship between the sine and cosine wave modulating parts.

Because $A^{2}(x, y)$ is an estimate of image power within the frequency passband of the wavelets, it can be a classifier of iris local textural appearance (e.g. fine or coarse). Emerging from the bottom of each module is a phase description $\phi(x, y)$ of the same local patch, as the arctangent of the ratio of the sine and cosine encoder field responses. Indeed the bits of the IrisCode are set on that basis [19], by specifying the quadrant (if phase is resolved to two bits) of the complex plane in which $\phi(x, y)$ resides for each patch of the $I(x, y)$ image. If phase is resolved more finely (or on a continuum), this combined description corresponds to the evolving phasor within Fig. 2. Extracting: (1) an amplitude function $A(x, y)$ useful for iris textural classification, and (2) a phase function $\phi(x, y)$ useful for iris identification, is arguably analogous to: (1) classifying a fingerprint as whorl, loop, or arch by its general flow pattern, and (2) actually identifying the fingerprint, by its detailed sequence of minutiae.

\section{METHODS AND DATABASES}

This paper examines, on databases larger than used in the papers cited above, various symmetry-related questions including whether genetically identical eyes have a biometric distance that is systematically smaller than it is between unrelated eyes. Without doubt, any algorithm that encoded iris colour or "general textural appearance" would find such similarities, as is obvious even from just visual inspection of the (visible wavelength) MZ eye images seen in Fig. 1. But such a finding is not expected for the standard phasebased IrisCode algorithm. This is an important matter for iris recognition, especially in large database searches and when deployed in populations having a high prevalence of genetic relatedness due to inbreeding (e.g. cousin marriages), as is the case in India where the entire population of 1.3 billion persons have had their IrisCodes enrolled.

One database used to probe this question was the University of Notre Dame Biometrics Database (henceforth NDBD) [20] [21], by computing the IrisCodes for 29,986 iris images acquired from 1,352 different eyes with an LG-4000 camera. All possible pairwise comparisons among these IrisCodes were performed, logging their Hamming distances into three disjoint sets: (1) comparisons just between the right and left eyes of each person; (2) comparisons between the eyes of different persons; and (3) comparisons between all same-eye images (not relevant here). This database was also used to look for symmetry within iris patterns, by creating mirrorflipped copies of them and comparing the resulting IrisCodes against the original ones. A further database consisting of iris images of twins, acquired at the Annual Festival of Beijing Twins Day, was made available by the Chinese Academy of Sciences (henceforth CASD) [7]. This database contains 3,183 images of 400 eyes (usually 10 images per eye) in 200 persons presenting as twins, thereby 100 pairs of twins, judged to be monozygotic by extreme similarity of their faces [7] but without DNA tests. We used these images to make all possible within-twin iris comparisons, distinguishing between ipsilateral $\left(\mathrm{R}_{1} \mathrm{R}_{2}\right.$ or $\left.\mathrm{L}_{1} \mathrm{~L}_{2}\right)$ and contralateral $\left(\mathrm{R}_{1} \mathrm{~L}_{2}\right.$ or $\left.\mathrm{L}_{1} \mathrm{R}_{2}\right)$ eye pairings in order to probe further questions of symmetry.

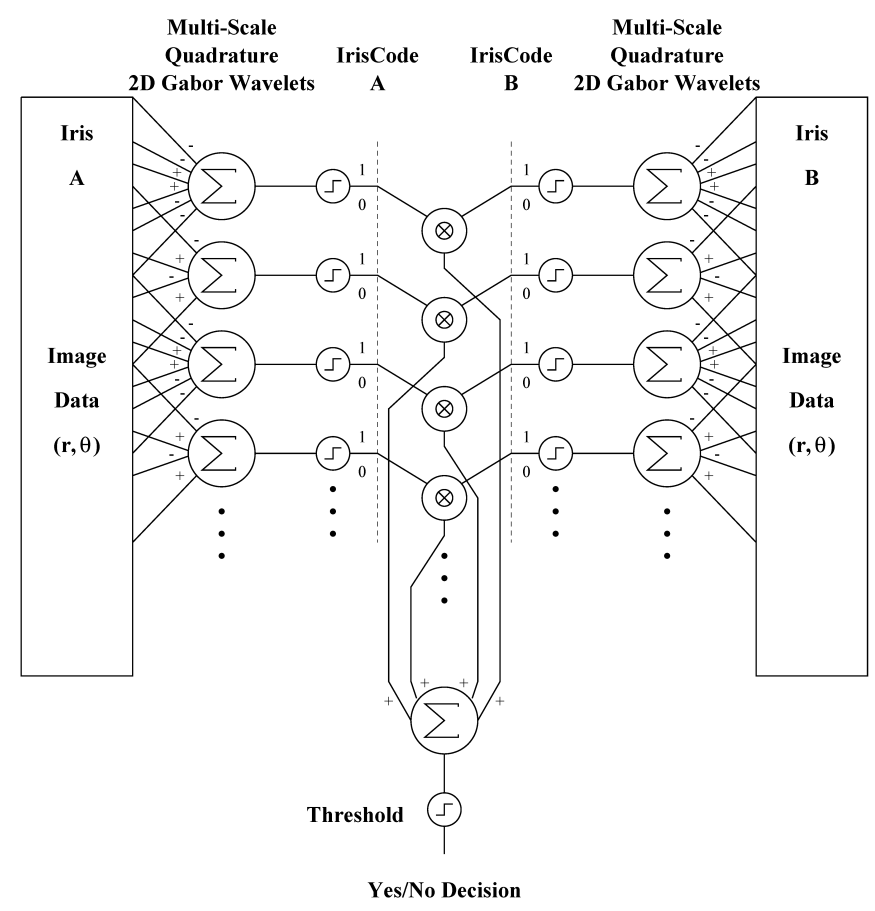

Fig. 3. Bit-parallel iris matching and search as a 'Convolutional Neural Net'.

The methods for encoding iris patterns into IrisCodes and then measuring their degree of similarity as a Hamming distance have been presented before [19] [22] and will not be rehearsed here in detail. Fig. 3 summarises these processes as the convolutional projections of two iris images (A and B) onto the wavelet encoder fields, symmetric and/or anti-symmetric, followed by thresholding of the resulting inner products $(\Sigma)$ for phase quantisation to set the bits of IrisCodes. These bit streams are then matched in parallel by Exclusive-OR comparison optimised over many candidate relative rotations, amounting to another, cyclic convolution, to discover a minimal scalar Hamming distance that enables a match decision. Today the algorithm [22] summarised by the network in Fig. 3 would be called a Convolutional Neural Net. Its underlying encoder fields, which are the Re part and the Im part (cosine or sine phase) elements of a complex 2D Gabor wavelet, are defined by local symmetry or anti-symmetry as was seen in Fig. 2. Thus at the very fundamental level of encoding iris discriminating information, the wavelet approach used in the standard algorithm is itself based on symmetries.

\section{RESULTS}

\section{A. Broken binocular symmetry}

The right and left eyes of a given person are genetically identical, of course. Previous studies on small databases [17] showed that their iris patterns are no more correlated than are 


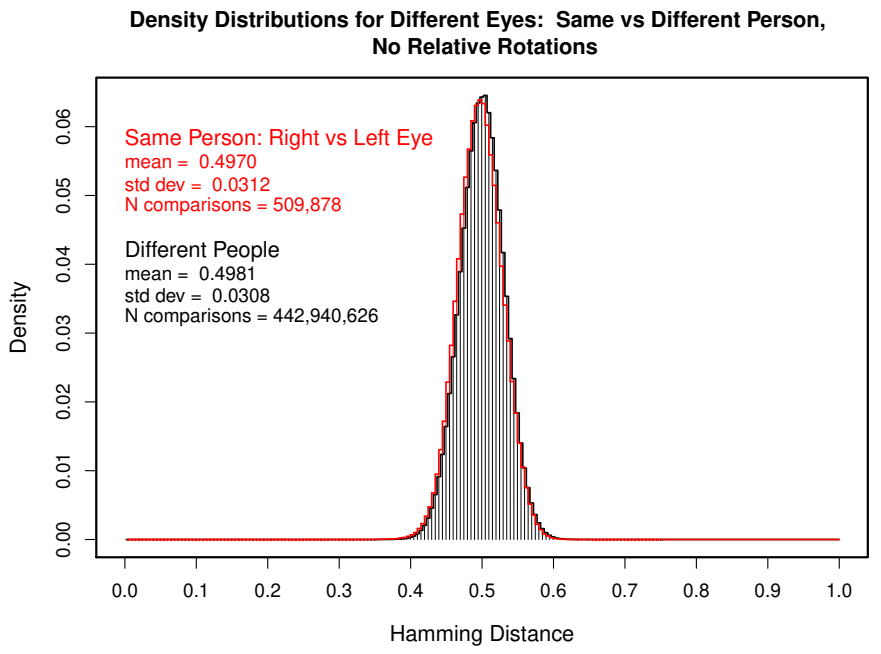

Fig. 4. Distributions of dissimilarity scores between individuals' two eyes, and between those of different people, without optimising for relative tilt.

those of genetically unrelated eyes, but we wished to confirm this "broken symmetry" using vastly more data. The NDBD allows nearly 443 million comparisons to be made between images of unrelated eyes, and nearly 510,000 comparisons to be made between images of a given person's two eyes. Fig. 4 presents together both those distributions of Hamming distance scores, when no relative rotations are employed to optimise the matching process. The two distributions appear indistinguishable. Their form is what would arise from tossing a fair coin 260 times in a 'run' and then tallying the fraction of (say) 'heads', in each of many such runs. In other words, both distributions in Fig. 4 are extremely well described as a $B(260,0.5)$ fractional binomial.

In Fig. 5 we present the two distributions that result after searching for the best match when allowing seven relative rotations, as performed in the standard algorithms because of uncertainty about head or camera tilt angle. Tallying the best (smallest) Hamming distance score in each such set of seven comparisons generates a new distribution with a smaller mean, nearer to 0.45 and whose functional form is readily derived [19] [22] as the minimal 'extreme value distribution' associated with multiple samples from $B(260,0.5)$. The two distributions in Fig. 5 are very well described by that form. Again, as was noted in Fig. 4, it is almost impossible to see a difference between the distributions for genetically identical (right, left) eyes versus unrelated eyes. There does not exist binocular symmetry in the iris patterns of a given person's two eyes, as encoded for purposes of iris recognition.

\section{B. Broken monozygotic twin symmetry}

In the preceding section comparing the two eyes of individuals, obviously all such comparisons were contralateral. $\mathrm{MZ}$ twins allow a richer exploration of genetically identical eyes. Using the same methods as above, we generated all possible comparisons between the eye images of paired twins among the 200 persons in the CASD database, with seven relative tilt angles to find each best match. This produced a total of 25,983 best match scores partioned into ipsilateral and

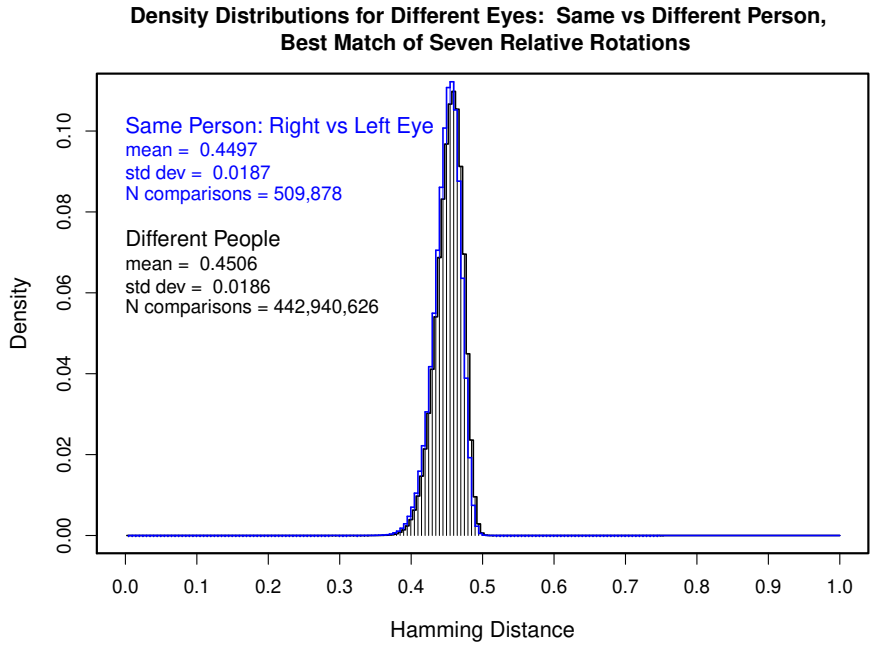

Fig. 5. Distributions of dissimilarity scores between individuals' two eyes, and between those of different people, after optimising for relative tilt.

contralateral comparisons between paired twins, plus 5 million other image comparisons. The best match scores for CASD twin comparisons, optimised over the relative tilt angles, are presented in Fig. 6 together with the distribution for such comparisons between unrelated eyes. As we saw in Fig. 5 for non-twins, these three distributions appear extremely similar to each other.



Fig. 6. Distributions of dissimilarity scores between twin ipsilateral eyes, twin contralateral eyes, and for unrelated eyes, optimising for relative tilt.

While Fig.s 4-6 show that for purposes of iris recognition, genetically identical eyes are essentially as distinguishable as are unrelated eyes, some very small differences between the density distributions are apparent. In order to determine whether these shifts have arisen by chance or whether they result from a more systematic effect, we performed a second set of analyses for each of the image databases. In these, we computed the average Hamming distance for each person in the database for each of several subsets: self contralateral eyes; twin pair ipsilateral eyes; twin pair contralateral eyes; unrelated ipsilateral eyes; and unrelated contralateral eyes. 
This partitioning allowed us to submit any two of these types of comparisons to a matched pairs t-test, which evaluates the hypothesis that the difference between them (computed for each person) is drawn from a distribution centered on zero.

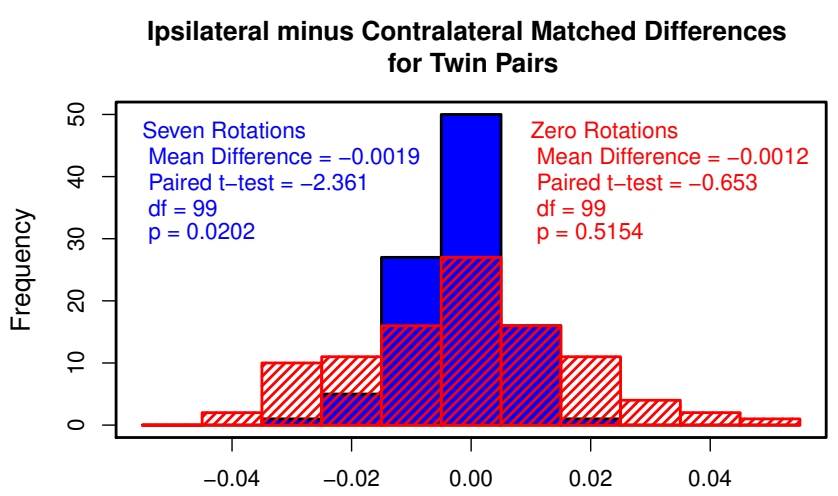

Difference between Mean Hamming Distance Scores

Self minus Unrelated Contralateral Matched Differences



Fig. 7. Distributions of differences in Hamming distances for eye pairings in twins, same person, and unrelated persons, ipsilaterally and contralaterally, to evaluate whether such differences are zero-centered or systematic.

This is a very sensitive test and there are large numbers of persons in each image set, so it is perhaps not surprising that we observed several statistically significant differences (meaning they are unlikely to have arisen by chance). The distributions of these differences are shown in Fig. 7 for these comparison cases in the CASD database. Twin pair ipsilateral eyes are statistically more similar to each other than twin pair contralateral eyes (mean difference -0.002), or to unrelated eyes (mean difference up to -0.003), after optimising for relative tilt angles. Statistically the eyes of a given person are more similar to each other than to either the ipsilateral or contralateral eyes of unrelated persons (mean differences about -0.002, also for NDBD). In each case, optimising over tilt angles reduces the variability in the difference scores, but even after the best rotated match is determined there remains a small systematic deviation from zero in the score differences. These reveal a systematic asymmetry between ipsilateral and contralateral eye pairings of twins, and also a difference in similarity between a given person's eyes, versus an unrelated contralateral eye. Kolmogorov-Smirnov tests also confirm the hypothesis that the distributions are different.

\section{Broken monocular mirror symmetry}

The final symmetry question that we explore here pertains to single eye images in isolation. We previously documented radial correlations [23] within iris patterns, by measuring how the internal mutual information depends on radial position and distance. We now investigate mirror-image symmetry within iris patterns. Using the NDBD iris database, we compared every image to itself after a flip around its vertical midline. This generated 29,798 similarity scores, for contrast against the 443 million comparisons between images of unrelated eyes presented earlier in Fig.s 4 and 5 using the same database. The results, first without optimising over multiple tilt angles, are presented in Fig. 8.



Fig. 8. Comparing a given eye image to itself after a simple mirror flip (red) doubles the variance of dissimilarity scores found for unrelated eyes (black).

The mean of the new distribution (red) remains very close to 0.5 , so on average, encoded iris images are no more similar to mirror-flipped copies of themselves than are images of different eyes. Thus monocular mirror symmetry is also absent. But strikingly there is almost a doubling, by a factor of 1.95, of the variance in the distribution of Hamming distance scores versus baseline. (The ratio of the standard deviations is 1.40 for the two distributions.) Although this has not been studied or reported previously, it is, in retrospect, quite understandable from the perspective of information theory. When an iris image is compared to itself after a flip, there is effectively only half as much entropy in this test as when two different iris images are compared. The reason is that once (say) the left half of an iris is compared to its flipped right half, then the remaining comparisons of the other halves are redundant with those already done. Essentially half an iris contains only half as much entropy as a full one. We would therefore expect that, whereas the $B(260,0.5)$ fractional binomial describes the usual distribution that we saw in Fig. 4 from comparing different eyes, instead now for mirrored images we would have a $B(130,0.5)$ fractional binomial. It should be recalled that whereas a binomial on the range of $[0, N]$ has variance proportional to $N$, namely $N p(1-p)$, a fractional binomial on the range $[0,1]$ has variance inversely proportional to $N$, namely $p(1-p) / N$. The general form of probability density 
function for $B(N, p)$ is $f(x)$ with $x=m / N \quad(0 \leq m \leq N)$ corresponding to an observed fractional Hamming distance:

$$
f(x)=\frac{N !}{m !(N-m) !} p^{m}(1-p)^{(N-m)}
$$

Because IrisCode matching involves a cyclic convolution corresponding to rotations of one eye relative to another to seek a best match (smallest $x$ ), we are in effect sampling $f(x)$ multiple times (say $k$ tilts) to create a new distribution of the resulting smallest scores. Obviously this new distribution will have a smaller mean than $p$ as can be seen in Fig. 9, both for the baseline comparisons between different people and for the comparisons between simply mirrored images. The new distribution after $k$ orientations of IrisCode comparisons still has a simple analytic form that can be derived theoretically.

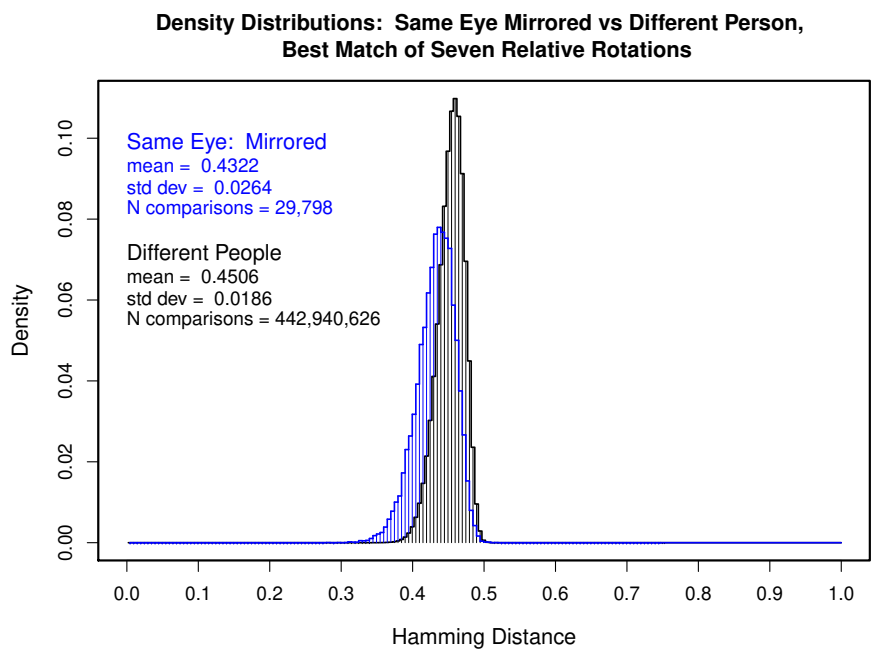

Fig. 9. Comparing a given eye image to itself after a simple mirror flip and optimising for tilt angles (blue) creates a different extreme value distribution than when comparing different eyes (black).

Let $f_{1}(x)$ be the density distribution obtained for Hamming distance scores between different IrisCodes when compared using a single tilt angle; for example, $f_{1}(x)$ might be the fractional binomial defined in Eqn (1). Its cumulative $F_{1}(x)$ is the probability of getting a score of $x$ or smaller:

$$
F_{1}(x)=\int_{0}^{x} f_{1}(x) d x
$$

or, equivalently,

$$
f_{1}(x)=\frac{d}{d x} F_{1}(x)
$$

The probability of not getting a score that is smaller than $x$ is therefore $1-F_{1}(x)$ in single comparisons, and it is $\left[1-F_{1}(x)\right]^{k}$ after carrying out $k$ such tests independently using $k$ different relative tilt angles. Thus the cumulative probability distribution $F_{k}(x)$ for observing scores of $x$ or smaller after optimising for relative orientation is

$$
F_{k}(x)=1-\left[1-F_{1}(x)\right]^{k}
$$

and the probability density distribution $f_{k}(x)$ expected for this cumulative is:

$$
\begin{aligned}
f_{k}(x) & =\frac{d}{d x} F_{k}(x) \\
& =k f_{1}(x)\left[1-F_{1}(x)\right]^{k-1}
\end{aligned}
$$

This theoretical extreme value distribution for the minimal score $x$ obtained after testing at $k$ relative orientations and assuming $f_{1}(x)=B(130,0.5)$ is plotted in Fig. 10, closely matching both of the observed distributions of mirror-image scores obtained for $k=1$ and $k=7$ relative orientations.

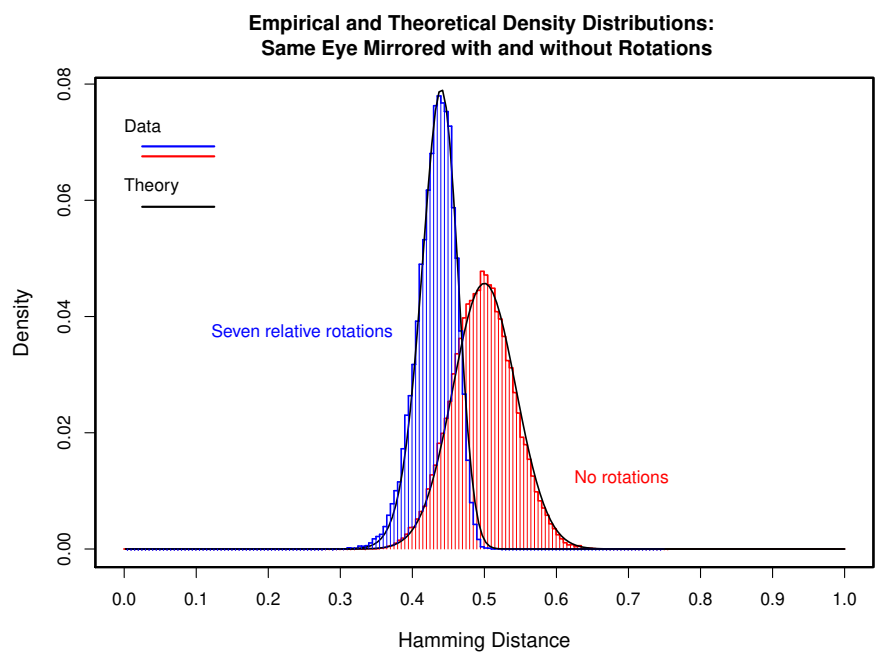

Fig. 10. The extreme value distribution (5) closely fits the observed match scores, for both $k=1$ and $k=7$ relative orientations. For mirrored image comparisons shown here the underlying binomial is $f_{1}(x)=B(130,0.5)$, but for normal comparisons as shown in Fig.s $4-5$ it is $B(260,0.5)$.

\section{DISCUSSION}

The broken symmetries studied in this paper confirmed earlier findings that the iris patterns of a given person, and also those of twins, are essentially as distinguishable as are those for unrelated eyes. This reflects the random morphogenesis of iris patterns in genetically identical phenotypes. But two new findings were unexpected: statistically significant differences between unrelated versus genetically identical eyes, whether in MZ twins or in a given person; and score variance doubling after monocular mirror flips. The latter finding, while novel, was easy to explain by assessment of entropy. But the former finding is open to both genetic and environmental hypotheses.

About $4 \%$ of light is reflected at an air-water interface, such as the moist surface of the cornea. In a bright outdoor environment, such as the Beijing annual Twins Day Festival, a visible structure in the surrounding environment may produce a bright ambient corneal reflection that is common to different eye images. Some corneal reflections are faintly visible at fixed positions in iris images in the CASD gallery, and such shared specular artefacts may be sufficient to produce the small reduction in Hamming distance scores by about 0.002 on average as highlighted in Fig. 7. It is likely that twin pairs would present in the same given time session, and likewise for when images of both of the eyes of a given person are 
acquired, so reflections of surrounding bright objects would be similar in such cases. This environmental effect may also contribute to differences in similarity observed for the NDBD gallery. Although acquired indoors, changes in environment or conditions (even if related only to windows or screens, given the $4 \%$ corneal reflectance rate) could lead to the apparent "template aging effect" [24], [25].

A twins-related hypothesis is that there really are some features in common in the ipsilateral eyes of MZ twins, as implied by Fig.s 6 and 7, despite the fact that the zygote splits within the first 12 days of conception. Intriguingly, by magnifying some of the twin eye images presented at the start of this paper in Fig. 1, we can see outlined in Fig. 11 some hints of such possible shared ipsilateral features in MZ twins. Given the faint analogy with 'quantum entanglement' of particles or photons emitted from a given quantum event and persisting over long distances, we may (archly) call this the QE hypothesis.
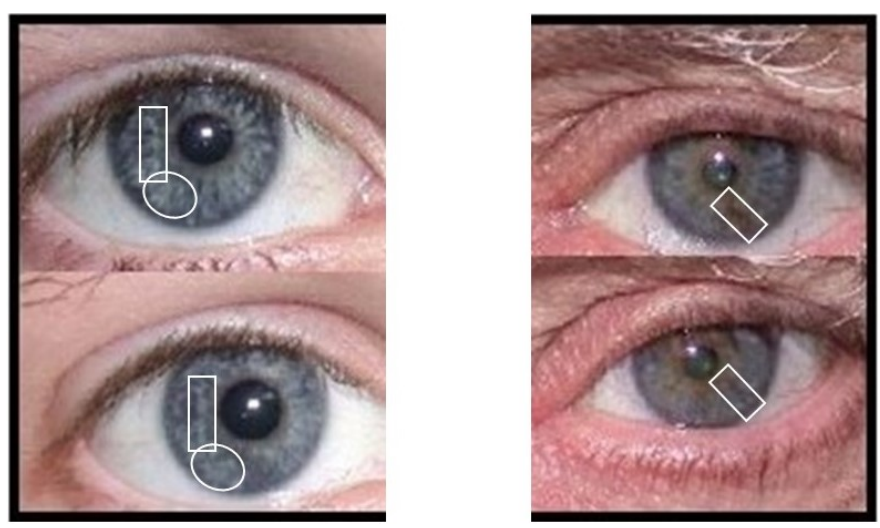

Fig. 11. Magnified portions of the MZ twin images shown in Fig. 1, with some shared ipsilateral features possibly supporting the QE hypothesis.

\section{CONCLUSiON}

Broken symmetries are critical for biometric discriminating power. If a person's two iris patterns were the same, or indeed if single iris patterns possessed mirror symmetry, then entropy would be lost and the impostors distribution would be broader (therefore less discriminating) as demonstrated in Fig. 8. It is remarkable both that encoded iris patterns exhibit so much random phenotypic complexity, and yet that the algorithm may detect a minute vestige of similarity in those developed from an identical genome. When IrisCodes are computed for genetically identical eyes, the developmental symmetries that human observers readily discern [13] are almost entirely broken; whereas such symmetries are only partially broken for $\mathrm{MZ}$ fingerprints [6], and for $\mathrm{MZ}$ faces they remain almost entirely intact [5]. Biometric distance arises from random or chaotic morphogenesis, and biometric discriminating entropy benefits from broken symmetries.

\section{ACKNOWLEDGEMENTS}

We are grateful to Richard Sturm and Mats Larsson of the Melanogenix Laboratory in Brisbane, Australia, for providing the visible-wavelength photographs of $\mathrm{MZ}$ twins presented in Fig. 1; and to the University of Notre Dame, and the Chinese Academy of Sciences, for making their Biometrics Databases available. Statistical analyses and generation of figures were performed using the ' $\mathrm{R}$ ' package: https://cran.r-project.org/

\section{REFERENCES}

[1] T. Cover and J. Thomas, Elements of Information Theory, 2nd ed. New York: Wiley-Interscience, 2006.

[2] B.H. Weber, D.J. Depew, J.D. Smith (eds), Entropy, Information, and Evolution. Cambridge: MIT Press, 1988.

[3] P.B. Green, C.S. Steele, S.C. Rennich, "Phyllotactic patterns: a biophysical mechanism for their origin", Annals of Botany 77 (5), 1996, pp. 515-528.

[4] S.L. Berger, T. Kouzarides, R. Shiekhattar, A. Shilatifard, "An operational definition of epigenetics", Genes \& Development 23 (7), 2009, pp. 781-783.

[5] P. Grother, M. Ngan, K. Hanaoka, "Ongoing Face Recognition Vendor Test (FRVT), Part 2: Identification”, NISTIR 8238, National Institute of Standards and Technology (Bethesda), 2018.

[6] A.K. Jain, S. Prabhakar, S. Pankanti, "On the similarity of identical twin fingerprints", Pattern Recognition 35 (11), 2002, pp. 2653-2663.

[7] Z. Sun, A.A. Paulino, J. Feng, Z. Chai, T. Tan, A.K. Jain, "A study of multibiometric traits of identical twins", Proc. SPIE 7667, Biometric Technology for Human Identification VII, (14 April 2010).

[8] S.N. Srihari, H. Srinivasan, G. Fang, "Discriminability of fingerprints of twins", Journal of Forensic Identification 58 (1), 2008, pp. 109-127.

[9] M. Pruitt, J. Grant, J. Paone, P. Flynn, R. Bruegge, "Facial recognition of identical twins", 2011 Int'l Joint Conf. on Biometrics (IJCB), Washington DC, pp. 1-8.

[10] J. Phillips, P. Flynn, K. Bowyer, R. Bruegge, P. Grother, G. Quinn, M. Pruitt. "Distinguishing identical twins by face recognition". In 2011 IEEE Conference on Automatic Face and Gesture Recognition and Workshops, Santa Barbara, CA, pp. 185-192.

[11] S. Biswas, K. Bowyer, P. Flynn, "A study of face recognition of identical twins by humans", 2011 Int'l Conf. on Information Forensics and Security, Iguacu Falls, Brazil pp. 1-6.

[12] M. Larsson, N. Pedersen, H. Stattin, "Importance of genetic effects for characteristics of the human iris", Twin Research and Human Genetics, 6 (3), 2003, pp. 192-200.

[13] K. Hollingsworth, K.W. Bowyer, P. Flynn, "Similarity of iris texture between identical twins", IEEE Conf. on Computer Vision and Pattern Recognition Workshops, 2010, pp. 22-29.

[14] D. Kennett, "Using genetic genealogy databases in missing persons cases and to develop suspect leads in violent crimes", Forensic Science International 301, 2019, pp. 107-117.

[15] R.A. Sturm, M. Larsson, "Genetics of human iris colour and patterns", Pigment Cell Melanoma Res. 22, 2009, pp. 544-562.

[16] E. Quillen, J. Guiltinan, S. Beleza, J. Rocha, R. Pereira, M. Shriver, "Iris texture traits show associations with iris color and genomic ancestry", Am. J. of Human Biology 23 (4), 2011, pp. 567-569.

[17] J. Daugman, C. Downing, "Epigenetic randomness, complexity and singularity of human iris patterns", Proc. Roy. Soc. 268 (1477), 2001, pp. 1737-1740.

[18] J. Daugman, C. Downing, "Demodulation, predictive coding, and spatial vision", Journal of the Optical Society of America A 12 (4), 1995, pp. 641-660.

[19] J. Daugman, "How iris recognition works", IEEE Trans. Circuits and Systems for Video Technology 14 (1), 2004, pp. 21-30.

[20] K.W. Bowyer, P.J. Flynn, "The ND-IRIS-0405 iris image dataset", 2016 in arXiv:1606.04853,2016-arxiv.org

[21] R. Connaughton, A. Sgroi, K. Bowyer, P. Flynn, "A multi-algorithmic analysis of three iris biometric sensors", IEEE Trans. Info. For. Sec. 7 (3), 2012, pp. 919-931.

[22] J. Daugman, "High confidence visual recognition of persons by a test of statistical independence", IEEE Trans. Pattern Anal. Mach. Intell. 15 (11), 1993, pp. 1148-1161.

[23] J. Daugman, C. Downing, "Radial correlations in iris patterns, and mutual information within IrisCodes", IET Biometrics 8 (3), 2019, pp. 185-189.

[24] S. Fenker, E. Ortiz, K. Bowyer, "Template aging phenomenon in iris recognition", IEEE Access 1, DOI: 10.1109/ACCESS.2013.2262916, 2013, pp. 266-274. 
[25] P. Grother, J. Matey, E. Tabassi, G. Quinn, M. Chumakov, "IREX VI: Temporal Stability of Iris Recognition Accuracy", NISTIR 7948, National Institute of Standards and Technology (Bethesda), 2013.

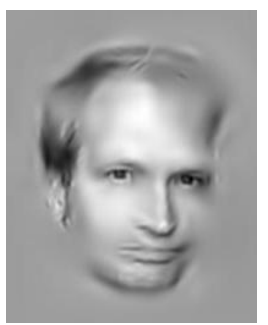

John Daugman received his degrees at Harvard University and then taught at Harvard before coming to Cambridge University, where he is Professor of Computer Vision and Pattern Recognition. He has held the Johann Bernoulli Chair of Mathematics and Informatics at the University of Groningen, and the Toshiba Endowed Chair at the Tokyo Institute of Technology. His areas of research and teaching at Cambridge include computer vision, information theory, neural computing, and statistical pattern recognition. Awards for his work in science and technology include the Information Technology Award and Medal of the British Computer Society, the "Time 100" Innovators Award, and the OBE, Order of the British Empire. He has been elected a Fellow of: the Royal Academy of Engineering; the US National Academy of Inventors; the Institute of Mathematics and its Applications; the British Computer Society; and he has been inducted into the US National Inventors Hall of Fame. He is the founder and benefactor of the Cambridge Chrysalis Trust. Here he is represented by a sparse sum of 2D Gabor wavelets in six orientations and five frequencies.



Cathryn Downing received her B.A. and her Ph.D. degrees at Yale University and Stanford University, respectively. Following post-doctoral appointments at New York University and at Harvard University, she became a Research Associate at Cambridge University. She is a Trustee of the Cambridge Chrysalis Trust. She and her co-author have published together on several subjects related to the present article including: demodulation coding; image quality metrics and predictive norms; radial correlations within iris patterns; the universality of the IrisCode impostors' distribution; and the effect of severe image compression on iris recognition. She is represented here by a linear combination of just several hundred local 2D Gabor wavelets having six orientations, two quadrature phases, and five frequencies, each an octave apart, thereby spanning four octaves in the same discrete self-similar log-polar system as in her co-author's photograph. 